\title{
A three-dimensional acoustics model using the method of fundamental solutions
}

\author{
Julieta António*, António Tadeu, Luís Godinho \\ Department of Civil Engineering, University of Coimbra, Polo II-Pinhal de Marrocos, P-3030-290 Coimbra, Portugal
}

Received 31 January 2007; accepted 10 October 2007

Available online 26 December 2007

\begin{abstract}
The method of fundamental solutions (MFS) is formulated in the frequency domain to model the sound wave propagation in threedimensional (3D) enclosed acoustic spaces. In this model the solution is obtained by approximation, using a linear combination of fundamental solutions for the $3 \mathrm{D}$ Helmholtz equation. Those solutions relate to a set of virtual sources placed over a surface placed outside the domain in order to avoid singularities. The materials coating the enclosed space surfaces can be assumed to be sound absorbent. This effect is introduced in the model by imposing impedance boundary conditions, with the impedance being defined as a function of the absorption coefficient. To impose these boundary conditions, a set of collocation points (observation points) needs to be selected along the boundary.

Time domain responses are obtained by applying an inverse Fourier transform to the former frequency domain results. In order to avoid "aliasing" phenomena in the time domain results, the computations introduce damping in the imaginary part of the frequency. This effect is later removed in the time domain by rescaling the response.

After corroborating the present solution against the analytical solution, known in closed form for the case of a parallelepiped room bounded by rigid walls, the model is used to solve the case of a dome.
\end{abstract}

(C) 2007 Elsevier Ltd. All rights reserved.

Keywords: MFS; Meshless; Acoustic model; Three-dimensional model

\section{Introduction}

The performance of rooms used for speech or music is highly influenced by the correct choice of a set of parameters during the design stage. The sound field produced inside enclosed spaces is dependent on their volume, geometry, coating materials, sound frequency, and occupancy. For this reason, the acoustics of rooms has been researched for many years in order to obtain models and experimental results that will be helpful to acoustic design. The modeling of the phenomena involved is not simple and different numerical methods of varying complexity have been developed.

There are classic statistical models, following the wellknown Sabine and Eyring theories that consider uniform energy density distribution, and recently some statistical

\footnotetext{
${ }^{*}$ Corresponding author.

E-mail address: julieta@dec.uc.pt (J. António).
}

models have been improved to include non-uniform reverberating energy density distribution [1].

Methods based on geometric acoustics are also widely used in room acoustics prediction. Among these methods is the image source method $[2,3]$ where the huge number of virtual sources required can be a limitation, and the ray tracing technique [4], valid in the high frequency range but including a degree of uncertainty since it is not sure that all the rays needed are included in the response. There are also hybrid methods combining those two [5].

Methods requiring domain discretization such as the finite element method (FEM), the finite difference method, and the boundary element method (BEM), have not been widely used to compute the propagation of sound, because of the high computation cost entailed. The FEM [6] and the finite difference method [7] fail because the domain under consideration has to be fully discretized, and very fine meshes are needed to solve excitations at high frequencies. Methods like the BEM [8] are more efficient 
in terms of computer cost as they only require the discretization of the boundaries, but they involve a large computational effort, particularly for very high frequencies.

Recently, several researchers have focused their work on meshless methods in order to avoid the time-consuming problem originated in mesh generation for complicated geometries. These methods have been used to solve some acoustic problems. The method of fundamental solutions (MFS) is applicable when a fundamental solution of the differential equation in question is known. Recent survey papers describe the method and various applications for it [9-11]. In Ref. [11], Fairweather et al. described and reviewed the MFS and related methods for the numerical solution of scattering and radiation problems in fluids and solids. Alves and Valtchev compared the plane waves method and the MFS for acoustic wave scattering [12]. Suleau and Bouillard applied the element-free Galerkin method to compute harmonic solutions of acoustic problems, governed by a Helmholtz equation [13]. Chen et al. employed the boundary collocation method using radial basis functions for the acoustic eigenanalysis of three-dimensional (3D) cavities [14]. In this paper, the MFS method is implemented to model a 3D acoustic problem. This method suffers from ill-conditioning of the system's linear equations, which is common when external source collocation methods are applied. Several techniques have been developed to handle the ill-conditioning of similar meshless collocation methods. Some use compactly supported radial basis functions [15], while others incorporate the least squares approach [16,17], apply a preconditioning technique [18] or use the matrix-free greedy algorithm [19].

Although the single value decomposition method (SVD) has traditionally been employed to solve ill-posed problems, in the case of the MFS, Chen et al. [20] demonstrated that the SVD is no more reliable than Gaussian elimination for non-noise boundary conditions. However, for noise boundary data, the truncated singular value decomposition method (TSVD) has been found to be more efficient than Gaussian elimination.

In this work, the sound field generated by a 3D sound source inside a 3D enclosure is modeled using the MFS. The model developed allows the boundaries to be rigid or absorbent and the final system of equations is solved using Gaussian elimination.

The problem is first formulated, the results are then validated using the image source method, and finally an application is presented.

\section{Problem formulation}

The pressure amplitude generated by a 3D source inside an air-filled 3D enclosed space is calculated by the MFS in the frequency domain $(\omega)$. The response inside the domain is found as a linear combination of fundamental solutions for the governing equation. Thus, the scattered pressure $(p)$ wave field is written as

$p=\sum_{s=1}^{N}\left[a_{s} G\left(\boldsymbol{x}, \boldsymbol{x}_{\boldsymbol{s}}, \omega\right)\right]$.

These solutions represent the sound field generated by a set of $N$ virtual sources with amplitude $a_{s}$, placed outside the domain on a fictitious boundary in order to avoid singularities. $G\left(\boldsymbol{x}, \boldsymbol{x}_{\boldsymbol{s}}, \omega\right)$ is the $3 \mathrm{D}$ Green's function for pressure, for a receiver placed at $\boldsymbol{x}$ with co-ordinates $(x, y, z)$, generated by pressure sources located at $\boldsymbol{x}_{\boldsymbol{s}}$ with co-ordinates $\left(x_{s}, y_{s}, z_{s}\right)$.

The 3D Green's function for pressure is well known

$G\left(x, x_{s}, \omega\right)=\frac{\mathrm{e}^{-\mathrm{i}(\omega / c) r}}{r}$,

where $r=\sqrt{\left(x-x_{s}\right)^{2}+\left(y-y_{s}\right)^{2}+\left(z-z_{s}\right)^{2}}, c$ is the sound wave velocity and $\mathrm{i}=\sqrt{-1}$. The coefficients $a_{s}$ are obtained by imposing the required boundary conditions at $M$ collocation points $\left(x_{k}, y_{k}, z_{k}\right)$ along the boundary. A system of $M$ equations by $N$ unknowns is then obtained. In this work, an equal number of collocation points and sources was considered, leading to a system $M \times M$. The resulting linear system was solved by Gaussian elimination [20].

For rigid enclosures, null velocities (incident velocity plus reflected velocity) are ascribed to the boundary. The Green's function for velocities is then given by

$H\left(\boldsymbol{x}_{s}, \boldsymbol{x}_{\boldsymbol{k}}, \omega, n\right)=-\frac{1}{\mathrm{i} \rho \omega} \frac{\partial G\left(\boldsymbol{x}, \boldsymbol{x}_{s}, \omega\right)}{\partial r} \frac{\partial r}{\partial n}$,

where $\rho$ is the air density and $n$ is the unit outward normal at the collocation point $\left(x_{k}, y_{k}, z_{k}\right)$. When the room's coating material is absorbent the governing equation is given by

$G\left(\boldsymbol{x}_{\boldsymbol{s}}, \boldsymbol{x}_{\boldsymbol{k}}, \omega\right)+\bar{Z} H\left(\boldsymbol{x}_{\boldsymbol{s}}, \boldsymbol{x}_{\boldsymbol{k}}, \omega, \boldsymbol{n}\right)=0$,

where $\hat{Z}$ is the material impedance given by the ratio between the pressure and velocity.

The material impedance can be expressed using the absorption coefficient $\alpha$ considering that $p_{r}=R p_{\text {inc }}, v_{r}=$ $-R v_{\text {inc }}, R=\sqrt{1-\alpha}$, where $R$ is the reflection coefficient, $p_{r}$ the reflected pressure, $p_{\text {inc }}$ the incident pressure, $v_{r}$ the reflected velocity, and $v_{\text {inc }}$ is the incident velocity. In fact, $\bar{Z}$ can be expressed as

$\widehat{Z}=\frac{p_{i n c}+R p_{i n c}}{v_{i n c}+R v_{i n c}}=\frac{p_{i n c}}{v_{i n c}}\left(\frac{1+\sqrt{1-\alpha}}{1-\sqrt{1-\alpha}}\right)$,

where $p_{\text {inc }}=\left(\mathrm{e}^{-\mathrm{i}(\omega / c) r} / r\right)$ and $v_{\text {inc }}=-\left([-\mathrm{i}(\omega / c) r-1] \mathrm{e}^{-\mathrm{i}(\omega / c) r)} /\right.$ $\left.\mathrm{i} \rho \omega r^{2}\right)(\partial r / \partial n)$.

In the case of an enclosure of arbitrary geometry built over a horizontal rigid base, the placement of collocation points at this surface can be avoided if an appropriate Green's function for a half-space is used:

$G\left(\boldsymbol{x}, \boldsymbol{x}_{\boldsymbol{k}}, \omega\right)=\frac{\mathrm{e}^{-\mathrm{i}(\omega / c) r}}{r}+\frac{\mathrm{e}^{-\mathrm{i}(\omega / c) r^{\prime}}}{r^{\prime}}$ 
with $r^{\prime}=\sqrt{\left(x-x_{s}\right)^{2}+\left(y-y_{s}\right)^{2}+\left(z+z_{s}\right)^{2}}$. The absorption may be ascribed, in a simplified way, to the base by assigning a reflection coefficient $R_{h}$ to it

$G\left(\boldsymbol{x}, \boldsymbol{x}_{\boldsymbol{k}}, \omega\right)=\frac{\mathrm{e}^{-\mathrm{i}(\omega / c) r}}{r}+R_{h} \frac{\mathrm{e}^{-\mathrm{i}(\omega / c) r^{\prime}}}{r^{\prime}}$.

The time domain responses are obtained applying an inverse Fourier transform to the frequency results. A finer sampling in the time domain is obtained by padding the frequency spectrum with zeros before applying the inverse discrete Fast Fourier Transform. The source amplitude follows a Ricker pulse shape. To prevent "aliasing" phenomena in the time domain, the calculations in the frequency domain are performed introducing a small amount of damping, $\omega_{c}=\omega-\mathrm{i} \eta . \quad \eta=0.7 \Delta \omega$ (with $\Delta \omega$ being the frequency increment) was chosen as the imaginary part of the angular frequency, to attenuate the wraparound by a factor of $\mathrm{e}^{0.7 \Delta \omega T}=81$, i.e., $38 \mathrm{~dB}$ (with $T=1 / \Delta \omega$ being the time window). This value of $\eta$ is commonly used in wave propagation analysis. This effect is later removed in the time domain by applying an exponential function $\mathrm{e}^{\eta t}$ [21].

The numerical implementation of this method is very simple since there is no need to solve integral equations, avoiding the integration of singularities that arise in other numerical methods. However, in this method, the number of virtual sources and their distance in relation to the boundaries are factors that influence the accuracy of the results.

A 3D model may require a large number of sources, and this number increases with the size of the space to be modeled and with the excitation frequency, as is usual with the traditional numerical methods (e.g., FEM and BEM). A huge number of virtual sources implies the generation of matrices of large dimensions, leading to a high computational cost or even making the application of the method of no practical use.

The application of the method will be limited to the modeling of small enclosures and to the calculation of not very high frequencies. Symmetry in the room's geometry should be used when possible to reduce the computational effort involved in solving the problem.

\section{Verification}

The MFS model developed for a 3D space is verified against the image source method [2], applied to a 3D rectangular rigid room $3 \mathrm{~m}$ wide, $3 \mathrm{~m}$ high and $4 \mathrm{~m}$ long, filled with air $\left(\rho=1.22 \mathrm{~kg} / \mathrm{m}^{3}\right.$ and $\left.c=340 \mathrm{~m} / \mathrm{s}\right)$. In this corroboration, the boundaries are assumed to be rigid (condition of null velocities).

The sound source is placed at $(0.5,0.5$, and $0.5 \mathrm{~m})$ and the pressure is registered at a receiver placed at $(1.5,2.0$, and $1.5 \mathrm{~m}$ ) (Fig. 1a).

In the MFS model, the response is calculated for different fixed distances between the fictitious and the real boundary. The number of sources and their positions in relation to the fictitious boundary influence the accuracy of the problem $[22,23]$. A boundary value problem converges as the sources are moved away from the boundary. Roundoff errors arise for large distances, however. The number of sources needs to be enough to represent the boundary

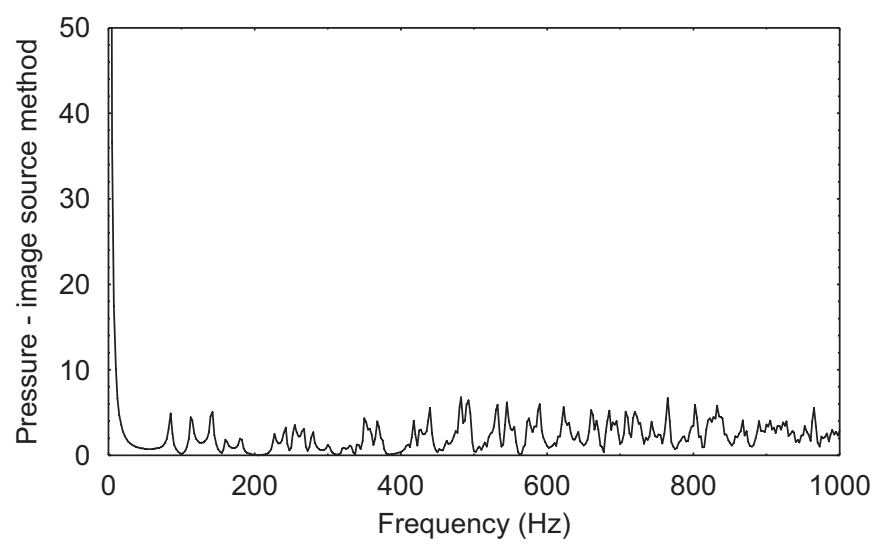

Fig. 2. Pressure amplitude obtained using the image source method.
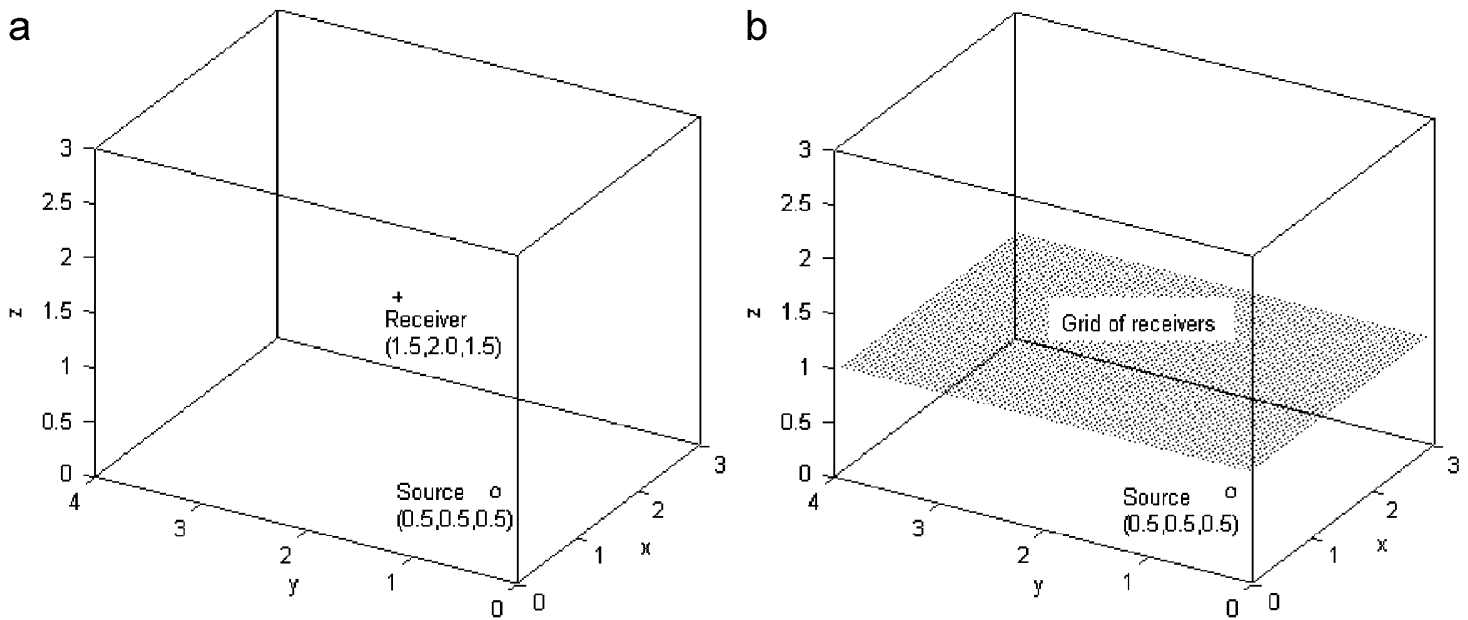

Fig. 1. Geometry of the problem and receiver's position: (a) receiver placed at (1.5, 2.0, and $1.5 \mathrm{~m}$ ); (b) grid of receivers. 
a

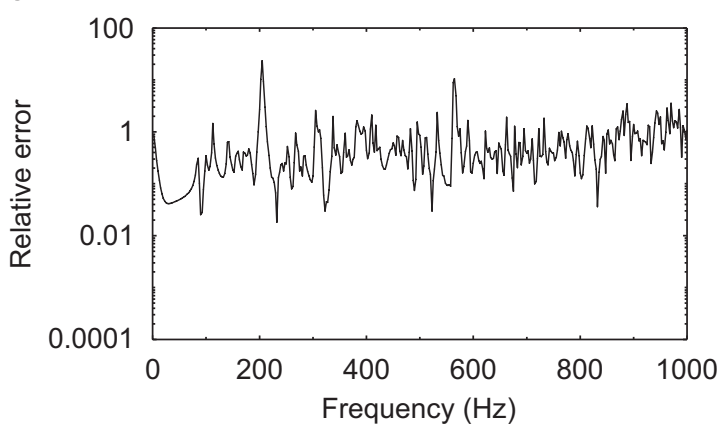

C

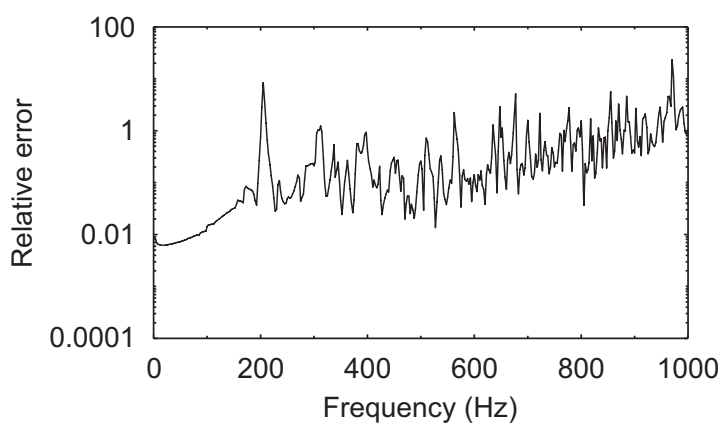

e

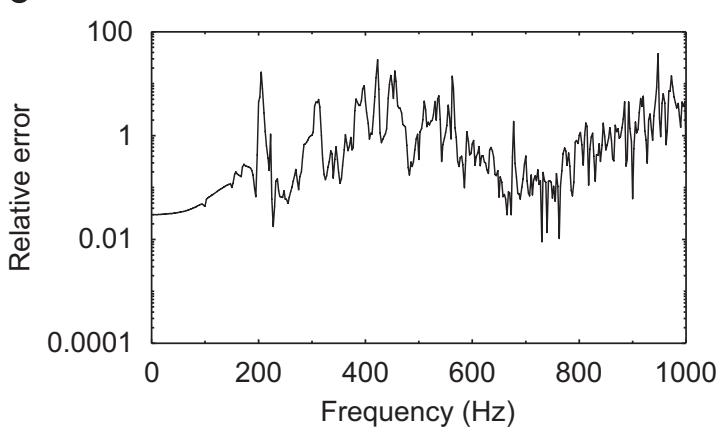

b

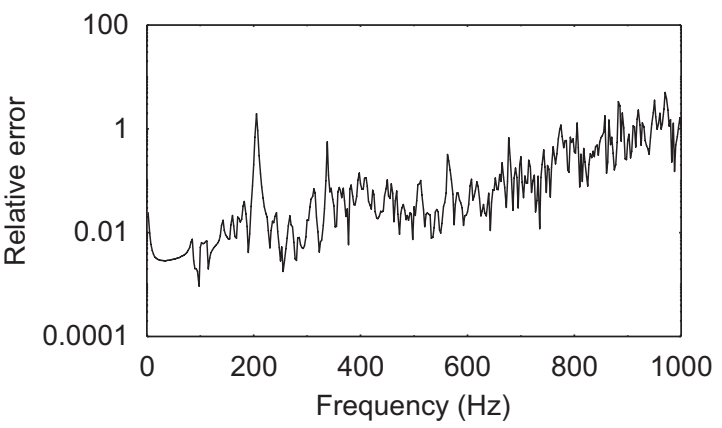

d

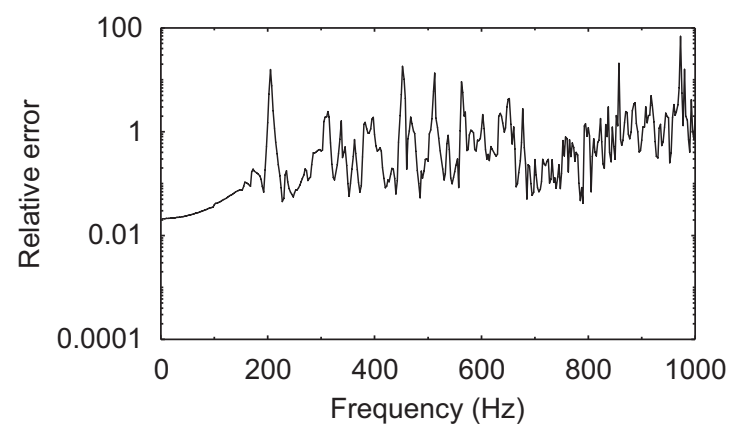

f

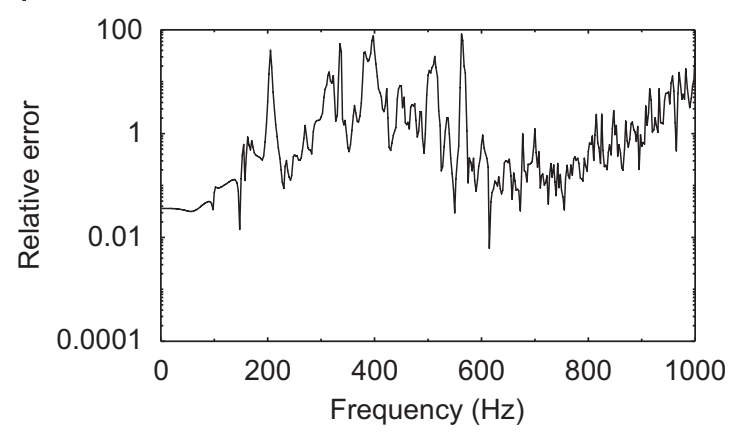

Fig. 3. Error obtained when the distances between the fictitious sources and the boundary, in the MFS model, are: (a) $0.1 \mathrm{~m}$; (b) $0.2 \mathrm{~m}$; (c) $0.3 \mathrm{~m}$; (d) $0.4 \mathrm{~m}$; (e) $0.5 \mathrm{~m}$; and (f) $0.6 \mathrm{~m}$.
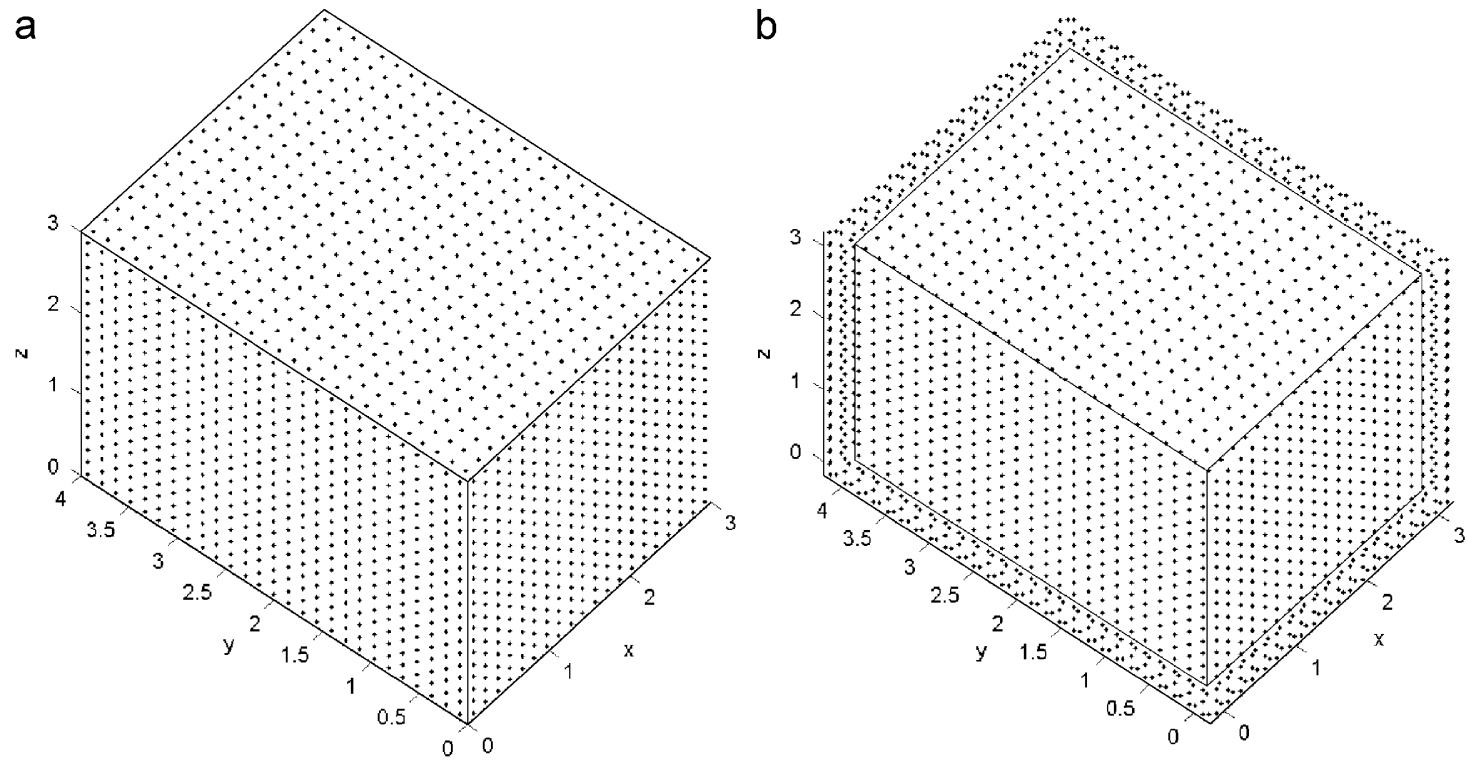

Fig. 4. Verification model: (a) collocation points and (b) virtual sources. 


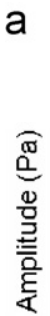

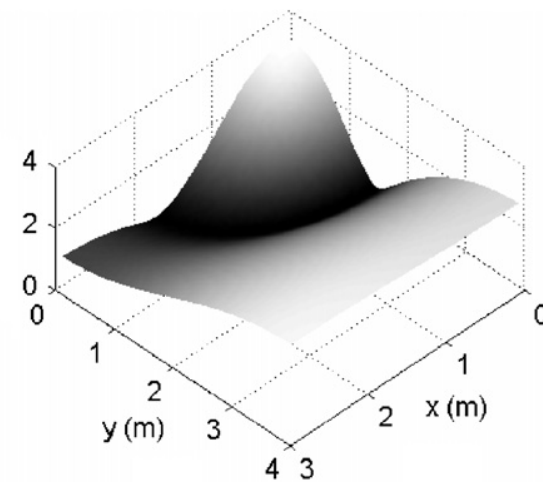

C

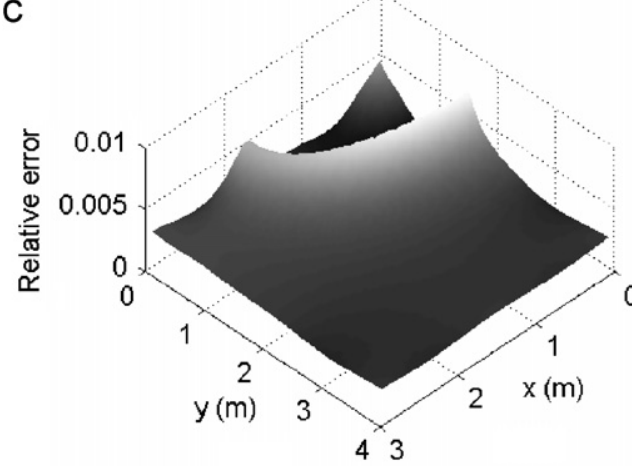

e

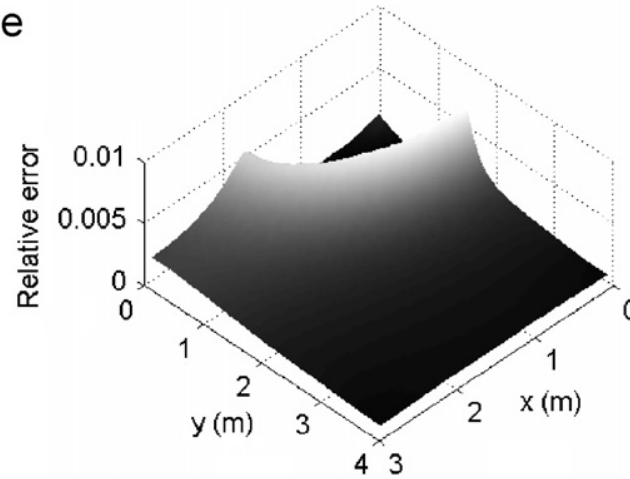

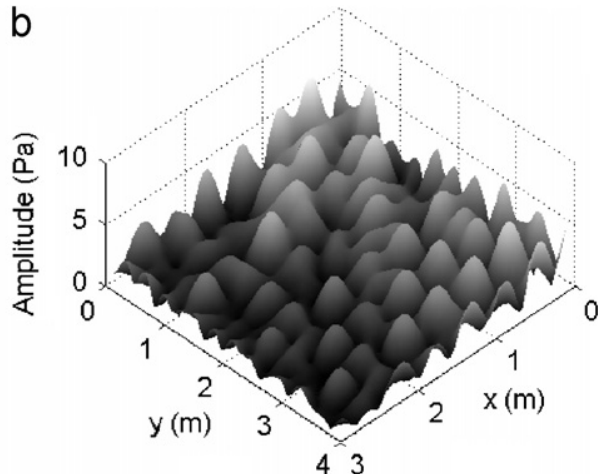

d

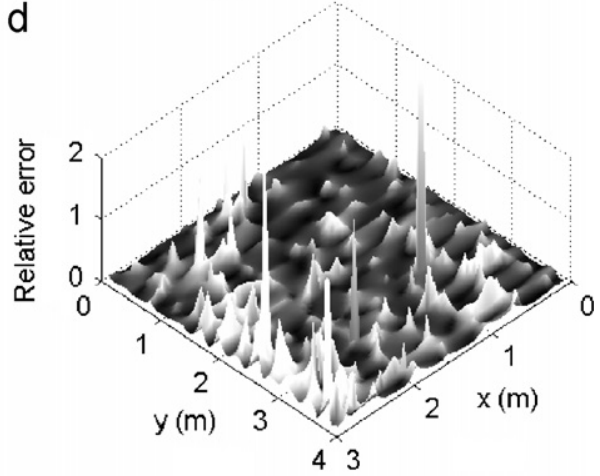

f

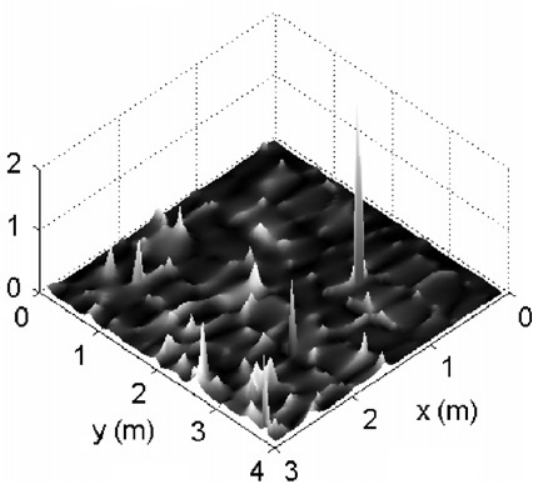

Fig. 5. Pressure amplitude registered at a grid of receivers using the image source method for frequencies (a) $25 \mathrm{~Hz}$ and (b) $600 \mathrm{~Hz}$; error obtained using the MFS with 1996 sources (c) $25 \mathrm{~Hz}$ and (d) $600 \mathrm{~Hz}$; error obtained using the MFS with 2960 sources (e) $25 \mathrm{~Hz}$ and (f) $600 \mathrm{~Hz}$.

conditions. Although extra sources lead to more accurate results, the ill-conditioning effects increase with more sources. In this example, different tests were carried out, revealing that the uniform distribution of sources over a fictitious spherical boundary would lead to high numerical errors. Better results have been achieved when the sources were distributed evenly at a fixed distance from the boundary. Accuracy depends, however, on the distance and on the number of sources. To illustrate this behavior, results obtained for two numbers of sources and for different positions of the fictitious boundary are now presented.

Six distances have been chosen for display: 0.1, 0.2, 0.3, 0.4, 0.5 , and $0.6 \mathrm{~m}$. The reference responses are those obtained with the image source model. In the MFS model, the number of virtual sources used for this verification is 1996.

Fig. 2 shows the pressure amplitude obtained using the image source method and Fig. 3 presents the error found

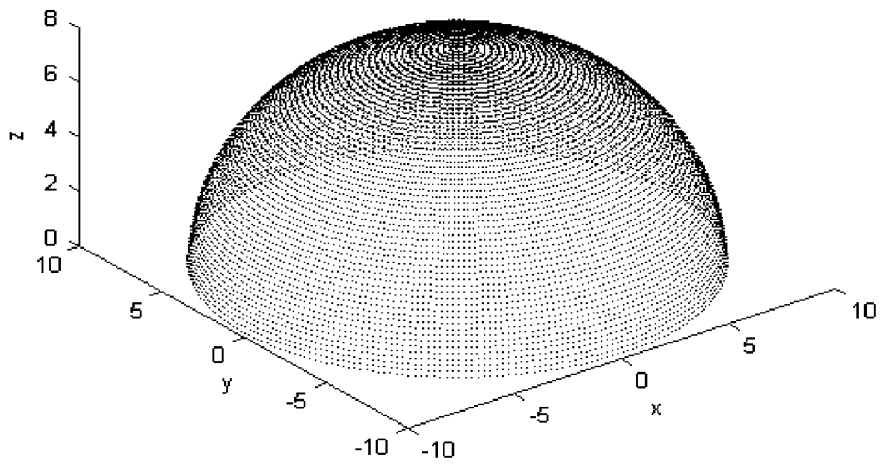

Fig. 6. Geometry of the problem.

with the MFS method. The error exhibits a significant variation with the distance between boundaries. It can be observed that the error is greater at high frequencies, which may signify that the number of sources and collocation 
points is insufficient at those frequencies. The best results are obtained when the distance between the fictitious and the real boundaries is set at $0.20 \mathrm{~m}$.

Calculations have also been performed for a grid of receivers $(59 \times 79$ spaced $0.05 \mathrm{~m})$, located at $z=1 \mathrm{~m}$ (Fig. 1b), for two distinct frequencies, 25 and $600 \mathrm{~Hz}$, and for the different fixed distances specified above. As before, the best results are achieved when the distance is set to $0.20 \mathrm{~m}$. Fig. 5 presents the absolute amplitude of the response obtained with the image source model and the error generated by the MFS when the fixed distance is $0.20 \mathrm{~m}$. Fig. $5 \mathrm{a}$ and $\mathrm{b}$ show the pressure amplitude for frequencies of 25 and $600 \mathrm{~Hz}$, respectively, while Fig. 5c and $d$ present the absolute error when using 1996 virtual sources and collocation points. Using the same distance to the boundary, the calculation is repeated increasing the number of virtual sources and collocation points to 2960 , as in Fig. 4. As Fig. 5e and $\mathrm{f}$ show, the error diminishes for a higher number of virtual sources and collocation points.

a
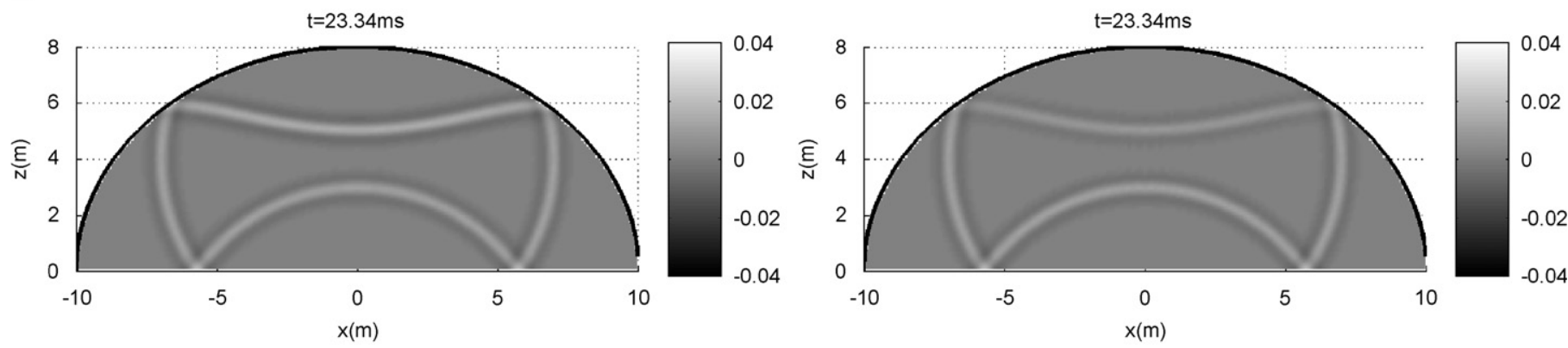

b
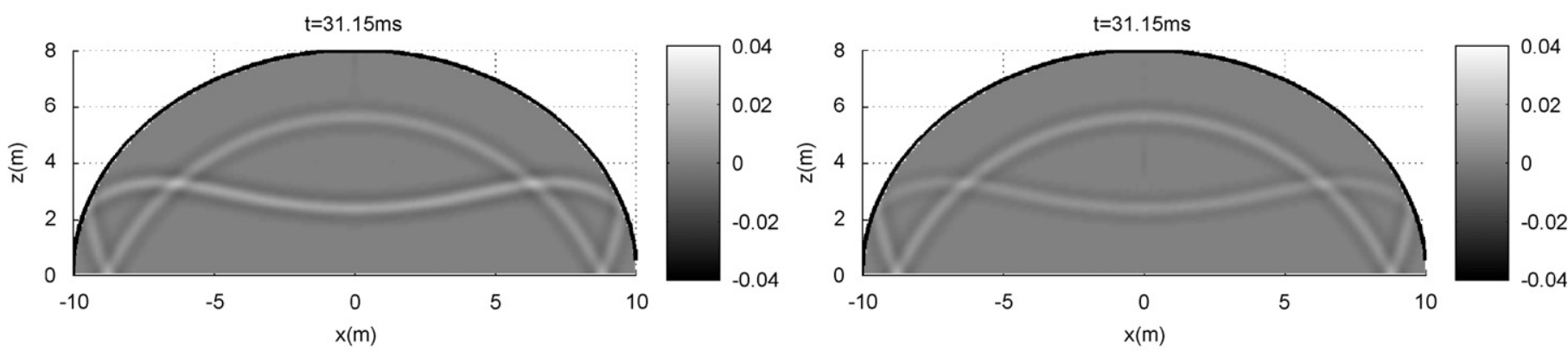

C
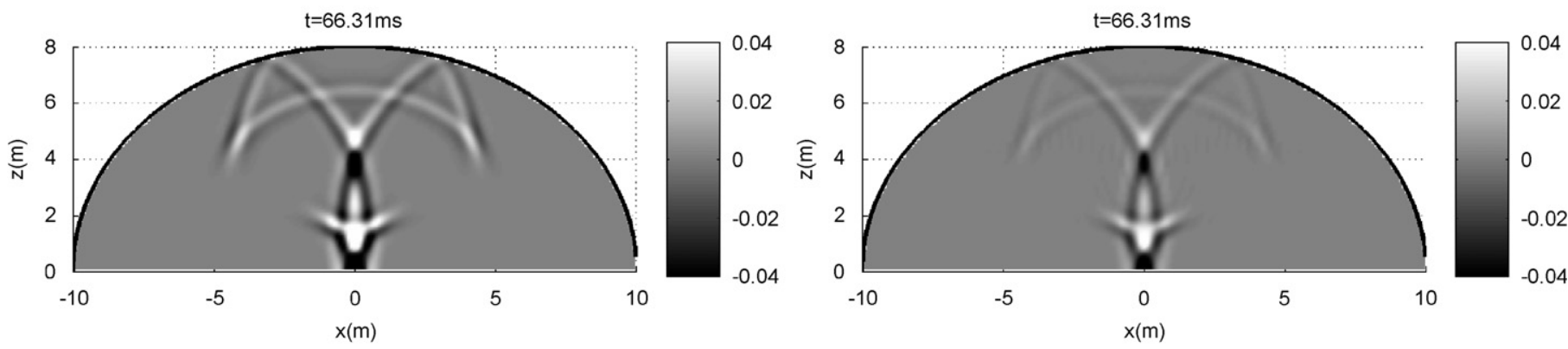

d
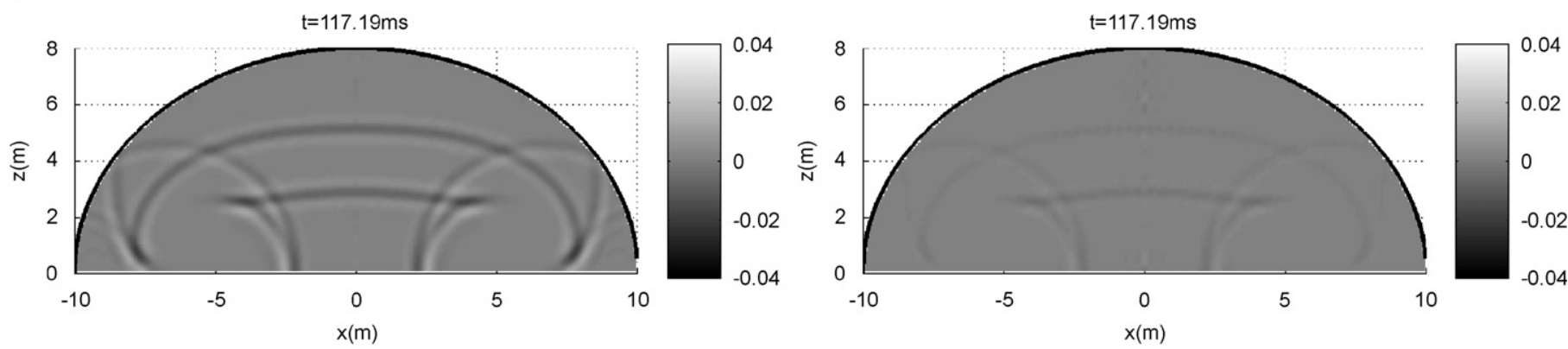

Fig. 7. Time displacements for a characteristic frequency of $350 \mathrm{~Hz}$, at a grid of receivers when the boundaries are rigid (left) and when the dome exhibits absorption (right): (a) $t=23.34 \mathrm{~ms}$; (b) $t=31.15 \mathrm{~ms}$; (c) $t=66.31 \mathrm{~ms}$; (d) $t=117.19 \mathrm{~ms}$. 


\section{Applications}

The algorithm described above is used to simulate the 3D wave field, generated inside a dome (with an oblate semi-ellipsoid shape) where the lengths of the three semiaxes are 10,10 , and $8 \mathrm{~m}$ in the $x, y$, and $z$ directions, respectively, as in Fig. 6. The air filling the 3D space has density $1.22 \mathrm{~kg} / \mathrm{m}^{3}$, allowing the propagation of sound waves whose velocity is $340 \mathrm{~m} / \mathrm{s}$. A 3D pressure source located at $(0.0,0.0$, and $4.0 \mathrm{~m})$ disturbs the medium, whose the pressure fluctuation is registered at a vertical grid of receivers placed at $x=0.0 \mathrm{~m}$. The receivers are $0.05 \mathrm{~m}$ apart in both directions ( $y$ and $z$ ).

Computations have been performed in the frequency domain from 2.5 to $1280 \mathrm{~Hz}$, with an increment of $2.5 \mathrm{~Hz}$. Pressure amplitudes in the frequency domain are submitted to an inverse Fourier transform in order to obtain responses in the time domain. The source is modeled as a Ricker pulse with a characteristic frequency of $350 \mathrm{~Hz}$. The number of sources and collocation points used is 16,141 . The fictitious sources were placed at $0.2 \mathrm{~m}$ from the boundary. Two situations are selected to illustrate the applicability of the model: case A-all the boundaries are rigid; case B-the pavement is rigid and the dome is absorbent.

A sequence of snapshots that displays the pressure wave field along the grid of receivers at different instants is presented. The pressure amplitude is displayed in a gray scale which ranges from black to white as the amplitude increases. Fig. 7 compares case A (left column) with case B (right column), where the absorption coefficient ascribed to the dome is $\alpha=0.7$. Both examples reveal a similar wave field pattern. However, when the dome is absorbent the wave amplitude suffers consecutive attenuations each time the waves reach the dome (Fig. 7).

\section{Conclusions}

An MFS algorithm using fundamental solutions for an acoustic half-space has been implemented in order to model wave propagation inside a room with a dome configuration. This model permits absorption to be prescribed at the boundaries. The accuracy of the results depends on the distance between the fictitious sources and boundaries and on the number of nodal points and sources. The appropriate choice of these two parameters yields reliable results.

In a $3 \mathrm{D}$ problem, the dimensions of the room and the computation frequency range are limited by the number of nodal points and sources required since they define the dimension of the system to be solved. It is expected that problems involving larger dimensions can be tackled using appropriate conditioning techniques of the resulting system matrix.

\section{References}

[1] Cotana F. An improved room acoustic model. Appl Acoust 2000;61: $1-25$.

[2] António J, Godinho L, Tadeu A. Reverberation times obtained using a numerical model versus those given by simplified formulas and measurements. Acta Acust. united Acust. 2002;88:252-61.

[3] Lee H, Lee B. An efficient algorithm for the image model technique. Appl Acoust 1988;24:87-115.

[4] Kulowski A. Algorithmic representation of the ray tracing technique. Appl Acoust 1985;18:449-69.

[5] Xiangyang Z, Ke-an C, Jincai S. Development of a hybrid computer model for simulating the complicated virtual sound field in enclosures. Appl Acoust 2002;63:481-91.

[6] Maluski S, Gibbs BM. The effect of construction material, contents and room geometry on the sound field in dwellings at low frequencies. Appl Acoust 2004;65(1):31-44.

[7] Savioja L, Rinne T, Takala T. Simulation of room acoustics with a 3D finite difference mesh. In: Proceedings of the ICMC'94, Aarhus, Denmark, 1994. p. 463-466.

[8] $\mathrm{Wu} \mathrm{TW}$, editor. Boundary element acoustics - fundamentals and computer codes. Southampton: WIT Press; 2000.

[9] Fairweather G, Karageorghis A. The method of fundamental solutions for elliptic boundary value problems. Adv Comput Math 1998;9:69-95.

[10] Golberg MA, Chen CS. The method of fundamental solutions for potential, Helmholtz and diffusion problems. Boundary Integral Methods: Numerical and Mathematical Aspects, Computational Engineering, vol. 1. Boston, MA: WIT Press/Computational Mechanics Publications; 1999. p. 103-176.

[11] Fairweather G, Karageorghis A, Martin PA. The method of fundamental solutions for scattering and radiation problems. Eng Anal Boundary Elem 2003;27:759-69.

[12] Alves CJS, Valtchev SS. Numerical comparison of two meshfree methods for acoustic wave scattering. Eng Anal Boundary Elem 2005;29:371-82.

[13] Suleau S, Bouillard $\mathrm{Ph}$. One-dimensional dispersion analysis for the element-free Galerkin method for the Helmholtz equation. Int $\mathbf{J}$ Numer Methods Eng 2000;47:1169-88.

[14] Chen JT, Chang MH, Chen KH, Chen IL. Boundary collocation method for acoustic eigenanalysis of three-dimensional cavities using radial basis function. Comput Mech 2002;29:392-408.

[15] Song K, Zhang X, Lu M. Meshless method based on collocation with consistent compactly supported radial basis functions. Acta Mech. Sin. 2004;20(5):551-7.

[16] Ling L, Kansa EJ. A least-squares preconditioner for radial basis functions collocation methods. Adv Comput Math 2005;23:31-54.

[17] Smyrlis Y-S. The method of fundamental solutions: a weighted leastsquares approach. BIT Numer Math 2006;46:163-94.

[18] Brown D, Ling L, Kansa E, Levesley J. On approximate cardinal preconditioning methods for solving PDEs with radial basis functions. Eng Anal Boundary Elem 2005;29:343-53.

[19] Hon YC, Schaback R, Zhou X. An adaptive greedy algorithm for solving large RBF collocation problems. Numer Algorithms 2003;32: $13-25$.

[20] Chen CS, Cho HA, Golberg MA. Some comments on the illconditioning of the method of fundamental solutions. Eng Anal Boundary Elem 2006;30:405-10.

[21] Kausel E, Roesset JM. Frequency domain analysis of undamped systems. J Eng Mech ASCE 1992;118(4):721-34.

[22] Fam G, Rashed Y. A study on the source points location in the method of fundamental solution. In: Proceedings on boundary elements, vol. 24. Sintra, Portugal, Southampton: WIT Press; 2002.

[23] Mitic P, Rashed YF. Convergence and stability of the method of meshless fundamental solutions using an array of randomly distributed sources. Eng Anal Boundary Elem 2004;28:143-53. 